\section{P4-S1.04 11C-CHOLINE SMALL ANIMAL PET IN EXPERIMENTAL CHLAMYDIA MURIDARUM INFECTION}

doi:10.1136/sextrans-2011-050108.506

${ }^{1} \mathrm{~A}$ Marangoni, ${ }^{2} \mathrm{C}$ Nanni, ${ }^{2} \mathrm{C}$ Quarta, ${ }^{1} \mathrm{R}$ Aldini, ${ }^{1} \mathrm{M}$ Donati, ${ }^{1} \mathrm{P}$ Nardini, ${ }^{1} \mathrm{C}$ Foschi, ${ }^{2} \mathrm{~S}$ Fanti, ${ }^{1} \mathrm{R}$ Cevenini. ${ }^{1}$ University of Bologna, Bologna, Italy; ${ }^{2}$ Azienda OspedalieroUniversitaria di Bologna, Policlinico S.Orsola-Malpighi, Italy)

Objectives Genital tract infection of female mice with Chlamydia muridarum closely mimics $C$ trachomatis acute genital tract infection in women. Aim of this study was to assess the predictivity of $11 \mathrm{C}$ Choline Small Animal PET for the identification of reactive animals and their follow-up at several times after vaginal challenge.

Methods Chlamydiae were grown in LLC-MK2 cells, cultivated in Eagle's MEM and elementary bodies were purified from cells by sucrose-gradients density centrifugation. Animals used in this study were 10 female Balb/c mice, 6-8 weeks old. All the animals received $2.5 \mathrm{mg}$ of medroxyprogesterone acetate intramuscular nine and 2 days prior the infection. Nine mice were infected by placing 106 IFUs of $C$ muridarum into the vaginal vault. Infection was induced under Ketamine anaesthesia. As control, one animal was challenged with SPG. At 5, 10 and 20 days after challenge mice underwent a 11C-Choline PET, as follows: each animal was anaesthetised with Sevofluorane 3-5\% oxygen $11 / \mathrm{min}$ and was injected with approximately $20 \mathrm{MBq}$ of $11 \mathrm{C}$-Choline, via the tail vein. The residual dose was measured to verify the effective dose injected. The animal was subsequently placed on the scanner bed in the prone position, after an uptake time of $5 \mathrm{~min}$. Images were acquired with a small animal PET tomograph (GE, eXplore Vista DR) for a total acquisition time of $20 \mathrm{~min}$. As the axial field of view was $4 \mathrm{~cm}$, one bed position was sufficient to cover the whole body. Images were reconstructed iteratively (OSEM 2D) and read in three planes (axial, sagittal and coronal). The scan was considered positive if areas of increased Choline uptake were present at sites consistent with the site of inflammation. The standard uptake value (SUV) was calculated by measuring the concentration of the labelled tracer in the region of interest and correcting it for mice body weight and injected dose. Infection was monitored by culturing cervical-vaginal secretions 6, 11 and 21 days after infection.

Results At 5 days, the mean vaginal SUV $( \pm S D$ ) of the cases was $0.83 \pm 0.39$, whereas the control showed a value of 0.278 . At 10 and 20 days, SUVs of the cases were $0.94 \pm 0.25$ and $1.17 \pm 0.21$, respectively, whereas SUV of the control was 0.299 and 0.302 , respectively. $C$ muridarum was isolated from all the infected animals during the study period.

Conclusions These preliminary data indicate that 11C-Choline PET could be a promising technique to in vivo evaluate inflammatory response to Chlamydia infection.

\section{P4-S1.05 POLYMERASE CHAIN REACTION-BASED TYPING OF PENA GENES EXHIBITING ELEVATED MIC VALUES TO CEPHALOSPORINS IN ISOLATES OF NEISSERIA GONORRHOEAE}

doi:10.1136/sextrans-2011-050108.507

${ }^{1} \mathrm{D}$ Trees, ${ }^{2} \mathrm{M}$ Burroughs, ${ }^{2} \mathrm{~S}$ Harris, ${ }^{2} \mathrm{~S}$ Johnson. ${ }^{1}$ Centers for Disease Control and Prevention, Atlanta, USA; ${ }^{2}$ CDC, USA

Objective Develop a PCR based assays for rapid analysis of isolates that exhibit elevated MIC values to penicillin and cephalosporins (MICs of $0.015-0.06 \mathrm{ug} / \mathrm{ml}$ to ceftriaxone). These primers were designed to detect mosaic and nonmosaic sequences in the pen $\mathrm{A}$ gene.

Methods Two sets of primer pairs were generated that allowed the detection of mosaic-type and nonmosaic-type penA gene sequences in isolates of $N$ gonorrhoeae that exhibited elevated MIC values to three cephalosporins. Additional primer sets were generated that detected the presence of an inserted aspartic acid residue in nonmosaic penA genes involved in resistance and to detect base substitutions to distinguish between two different mosaic penA genes. DNA sequences were determined as necessary by standard sequencing or pyrosequencing.

Results PCR analysis of 28 isolates, exhibiting elevated MIC values to cephalosporins and from different geographic areas, that used these primer sets in combination allowed for the detection of mosaic penA genes in 12 isolates. The remaining isolates possessed nonmosaic genes. Not all of the 12 isolates contained complete mosaic-type sequences with five of the isolates indicating mosaic sequences present in the $5^{\prime}$ region of the penA gene with wild type DNA sequence replacing the last $120 \mathrm{bp}$ of the gene. Another isolate contained nonmosaic penA sequences with an aspartic acid insertion (after aa 345) in the $5^{\prime}$ region of the gene but mosaic sequences in the $3^{\prime}$ end. Finally, mosaic-related sequences were detected by PCR and confirmed by DNA sequencing in the last 106 base pairs of the penA gene of a penicillin sensitive strain isolated prior to 1976 .

Conclusion PCR can be used to easily detect the presence of mosaic penA genes in isolates of $N$ gonorrhoeae. This study has shown the 3 region of the gene may contain mosaic, partial mosaic and nonmosaic-type sequences and that mosaic-type sequence has been encountered in the $3^{\prime}$ region of penA in a sensitive strain. We have also observed isolates with increased MICs to cephalosporins that do not contain mosaic sequences. Finally, isolates that possess mosaic penA genes have been detected in dispersed geographic regions in the US, which is of concern due to the evidence mosaictype penA alleles are involved in the process of increasing gonococcal MICs to cephalosporins.

\section{P4-S1.06 HIGH-RESOLUTION MULTILOCUS SEQUENCE TYPING (MLST) OF CHLAMYDIA TRACHOMATIS FROM THREE CATCHMENT AREAS IN NORTH AND CENTRAL NORWAY}

doi:10.1136/sextrans-2011-050108.508

${ }^{1} \mathrm{~B}$ Herrmann, ${ }^{1} \mathrm{~L}$ Christerson, ${ }^{2} \mathrm{~K}$ Gravningen, ${ }^{1} \mathrm{~A}$ Stahlsten, ${ }^{1} \mathrm{~K}$ Ödman, ${ }^{1} \mathrm{D}$ Nguyen, ${ }^{1} \mathrm{~J}$ Isaksson, ${ }^{2} \mathrm{~A}$ S Furberg, ${ }^{3} \mathrm{G}$ S Simonsen. ${ }^{1}$ Uppsala University, Uppsala, Sweden; ${ }^{2}$ University of Tromso, Tromso, Norway; ${ }^{3}$ University Hospital of North Norway, Tromso, Norway

Background The $C$ trachomatis incidence rate in the county of Finnmark in North Norway is twice as high as in the rest of the country. This study genetically characterises the $C$ trachomatis strains in Finnmark and compares them with strains from two other counties in Norway.

Methods DNA sequencing of ompA, which encodes the major outer membrane protein MOMP, and a high-resolution multilocus sequence typing (MLST) system, which is based on PCR amplification and DNA sequencing of five highly variable genetic regions (not housekeeping genes as in conventional MLST), were used. In total, 80 specimens from adolescents mainly aged 15-19 were collected in five high schools $(n=60)$ and in the laboratory routine $(n=20)$ in Finnmark (extended county with sparse population in minor municipalities) and compared to 80 laboratory specimens from Tromsø county (capital of North Norway) and 88 laboratory specimens from Trondheim in Central Norway.

Results Sequencing of ompA detected 11 genotypes in these 248 specimens. The MLST analysis displayed 50 sequence types (STs), providing five times higher resolution than ompA. Thirty-one (62\%) of the STs were novel. The study revealed overlapping STs between the different counties, but $64 \%$ of the STs were unique for specific counties (Abstract P4-S1.06 figure 1). The MLST genetic diversity was lower in Finnmark (0.26 ST per specimen compared to 0.34 in Troms $\varnothing$ and 0.32 in Trondheim, respectively), but the geographical 